Pacific Journal of Mathematics

ON MINIMAL AND MAXIMAL EIGENVALUE GAPS AND 


\section{ON MINIMAL AND MAXIMAL EIGENVALUE GAPS AND THEIR CAUSES}

Mark S. Ashbaugh, Evans M. Harrell, II, ANd Roman Svirsky

We consider quantum-mechanical potentials giving rise to minimal (or maximal) eigenvalue gaps subject to $L^{p}$ constraints in $n$ dimensions. We prove existence and characterization theorems for optimizing potentials. The tunneling effect through a single barrier is shown always to be the cause of minimal gaps, and in some cases the gap minimizers are shown to be specific double-well potentials.

I. Introduction. Let $\Omega$ be a bounded, smooth domain in $\mathscr{R}^{n}$, and consider the Schrödinger operator

$$
H=-\Delta+V(x)
$$

acting on $L^{2}(\Omega)$, with zero Dirichlet boundary conditions. As is well known, for reasonable potentials $V$ the spectrum consists of eigenvalues $\left\{E_{i}\right\}$, conventionally numbered in an increasing sequence,

$$
-\infty<E_{1}<E_{2} \leq E_{3} \leq \cdots .
$$

The eigenvalues correspond to the energy levels, in atomic units, of a quantum particle in the potential energy $V$, imagined as $+\infty$ outside $\Omega$. We refer to $E_{1}$ as the ground state, $E_{2}$ as the first excited state, and

$$
\Gamma=E_{2}-E_{1}
$$

as the fundamental gap.

Bounds on the fundamental gap have been the subject of a number of recent works [1], [23], [25], usually with assumptions imposed on both $V$ and $\Omega$ that can loosely be characterized as convex. If $R$ is a characteristic diameter of the problem, then with these assumptions $\Gamma$ can be no smaller than const. $R^{-2}$. Without the convex assumptions, on the other hand, exponentially small fundamental gaps $\Gamma=O(\exp (-$ const. $R))$, are known to arise in double-well problems, owing to the tunneling effect, and also in problems on pinched or dumbell-shaped domains [4], [19]. Recently, a pair of papers by Kirsch and Simon [14], [15] established, roughly, that the fundamental gap is bounded below by a polynomial in $R$ times $\exp (-$ const. $R)$, 
although with constants that differ from the tunneling constants. Related work appears in [13], [17].

The purpose of this article is to investigate mechanisms causing exponentially small gaps, and, in particular, to provide evidence that the smallest possible gaps are due to double-well type tunneling. The approach we follow is one of direct optimization, in the spirit of earlier papers on other spectral properties [2], [3], [8], [11], [24], and especially [9], where more discussion of the fundamental gap and some initial investigation along these lines are to be found.

The analogous problem of maximizing the fundamental gap does not seem to have as much physical importance as that of minimizing it. It is, however, intriguing as a mathematical problem in its own right, and is treated here by similar methods.

In $\S$ II we use straightforward compactness arguments to establish the existence of potentials $V$ that optimize the fundamental gap subject to $L^{p}$ constraints on $V$, and then show how to characterize them by direct optimization. This entails some delicate perturbation theory. In particular, we derive "switching principles" relating the high and low parts of the optimal potentials to the ratio of the corresponding eigenfunctions, and deduce that minimal gaps are caused by tunneling through a single barrier, in the sense that the positive support of a gapminimizing potential will be shown to be a connected set. Speaking pictorially, gap-minimizing potentials have no "islands." Analogously, unless $E_{2}$ is degenerate, gap-maximizing potentials have no "lakes." The main results are stated in Theorems II.3-II.6. In the third section we analyze the one-dimensional case, where the gap minimizers are shown to be symmetric double wells.

II. Existence and characterization of optimizing potentials. We consider the differential eigenvalue equation

$$
H \psi:=(-\Delta+V(x)) \psi=E \psi
$$

on a connected, bounded, open domain $\Omega \subset \mathscr{R}^{n}$ with a smooth boundary and Dirichlet boundary conditions. The function $V$, referred to as the potential, is real-valued and measurable, and further conditions will always be imposed making $H$ self-adjoint. We consider the fundamental gap $\Gamma$ of $(1.2)$ as a functional on potentials belonging to one of the classes

$$
S_{p, M}(\Omega):=\left\{V: \operatorname{supp} V \subset \Omega,\|V\|_{p} \leq M\right\}
$$

for some fixed $M \geq 0$ and $p>n / 2$ when $n \geq 2$. When $n=1$ we 
consider all $p>1$ as in (2.2) and in addition by convention set

$$
\begin{aligned}
S_{1, M}(\Omega):= & \{\text { the bounded real Borel measures } \\
& \text { of total Mass } M \text { supported in } \Omega\} .
\end{aligned}
$$

We begin with a discussion of the existence of optimal potentials. It is known that for any of these classes $V$ is relatively form bounded, and therefore $H$ is self-adjoint with the quadratic-form domain $W_{0}^{1,2}$, (1.1) holds, and $E_{1}$ is nondegenerate with a positive eigenfunction [7], [20], [21]. Moreover, let $E_{j}(V)$ denote the $j$ th eigenvalue of $H$ as defined by the min-max principle. Then $E_{j}(V)$ is bounded uniformly from above and below on $S_{p, M}(\Omega)$ for any fixed $p, M$ as specified above. (See [6], where this is proved for $j=1$; the generalization to higher eigenvalues is straightforward.) Obviously, the functional $\Gamma(V)=E_{2}-E_{1}$ is likewise uniformly bounded.

The key to proving the existence of optimizers is the well-known Rellich-Kondrashov Embedding Theorem [7], which states that the Sobolev space $W_{0}^{t, p}$ is compactly embedded in $L^{q}$ for $t p<n, q<$ $n p /(n-t p)$ and in $C^{m}(\bar{\Omega})$ for $0 \leq m<t-n / p$. The technique is fairly standard.

THEOREM II.1. Let $\Omega$ and $H$ be as above and fix $M, p$. Then the functional $\Gamma(V)$ attains its maximum and minimum on $S_{p, M}(\Omega)$.

Proof. We shall prove only the existence of the minimizer $V^{\sharp}$ such that $\Gamma\left(V^{\sharp}\right)=\Gamma^{\sharp}:=\inf _{S_{p, M}} \Gamma(V)$. The existence of a maximizer is entirely similar.

Let $\left\langle V^{(k)}, \psi_{j}^{(k)}, E_{j}^{(k)}\right\rangle, j=1,2, k=1,2, \ldots$, be a sequence of potentials in $S_{p, M}$, eigenfunctions normalized in $W_{0}^{1,2}$, and eigenvalues such that $\lim _{k} \Gamma\left(V^{(k)}\right) \rightarrow \inf \Gamma(V)$. By passing to subsequences, we can assume that $E_{j}^{(k)} \rightarrow E_{j}^{\sharp}, \Gamma\left(V^{(k)}\right) \rightarrow \Gamma^{\sharp}$, and that $V^{(k)}$ tends to a limit $V^{\sharp}$ in the weak $L^{p}$ sense (or weak-* sense in the case $S_{1, M}$ ).

We claim that we can extract a uniformly convergent subsequence from $\left\{\psi_{j}^{(k)}\right\}$. This is immediate for $n=1$, for in this case $W_{0}^{1,2}$ is compactly embedded in $C^{0}(\bar{\Omega})$ by the Rellich-Kondrashov Theorem. For $n \geq 3$ the Sobolev inequality

$$
\left\|\psi_{j}^{(k)}\right\|_{2 n /(n-2)} \leq C\left\|\psi_{j}^{(k)}\right\|_{W_{0}^{1,2}},
$$


where the constant $C$ depends only on $n$ and $p$, tells us that $\left\{\psi_{j}^{(k)}\right\}$ is bounded in $L^{q}$ for $q=2 n /(n-2)$. For $n=2$ it is bounded in $L^{q}$ for all $q<\infty$.

Thus from

$$
\Delta \psi_{j}^{(k)}=\left(V^{(k)}-E_{j}^{(k)}\right) \psi_{j}^{(k)}
$$

we find with the help of Hölder's inequality that $\left\{\Delta \psi_{j}^{(k)}\right\}$ is bounded in $L^{r}$, where

$$
\frac{1}{r}=\frac{1}{p}+\frac{1}{q} \text { and } r>\frac{2 n}{n+2} \geq 1
$$

for $n \geq 2$. Therefore $\left\{\psi_{j}^{(k)}\right\}$ is bounded in $W_{0}^{2, r}$. For $n=2$, $1<r<p$, but can be chosen arbitrarily close to $p>n / 2$, and we conclude from the Rellich-Kondrashov Theorem that the set $\left\{\psi_{j}^{(k)}\right\}$ is compactly embedded in $C^{0}(\bar{\Omega})$. For $n \geq 3$, the Sobolev inequality implies that $\left\{\psi_{j}^{(k)}\right\}$ is bounded in $L^{s}$ with $s=n r /(n-2 r)>q$. After a finite number of repetitions of the above argument we discover that $\left\{\psi_{j}^{(k)}\right\}$ is bounded in a space with a high enough index to be compactly embedded in $C^{0}(\overline{\boldsymbol{\Omega}})$.

We now renormalize $\left\{\psi_{j}^{(k)}\right\}$ in the $L^{\infty}$-norm and observe from the Schrödinger equation that $\left\{\psi_{j}^{(k)}\right\}$ is a bounded set in $W_{0}^{2, p}$ which is compactly embedded into $C^{0}(\bar{\Omega})$ since $2 p>n$. We denote the limit $\psi_{j}^{\sharp}$ and observe that it is not identically zero.

It is now easy to see from the eigenvalue equations

$$
\left(-\Delta+V^{(k)}\right) \psi_{i}^{(k)}=E_{i}^{(k)}
$$

that

$$
\left(-\Delta+V^{\sharp}\right) \psi_{i}^{\sharp}=E_{i}^{\sharp} \psi_{i}^{\sharp}
$$

in the sense of distributions. We finally observe that $E_{2}^{\sharp} \neq E_{1}^{\sharp}$, since the ground-state eigenvalue is nondegenerate, so $\Gamma\left(V^{\sharp}\right)=\Gamma^{\sharp}>0$.

A complementary point of view can also be adopted. Since eigenvalues change continuously under perturbations $V \rightarrow V+\kappa P$, with $P$ bounded, $\Gamma$ is a continuous monotonic function of $M$ for each fixed $p>n / 2$ (resp. $p \geq 1$ ). Hence, given a positive real number $\Gamma<\Gamma(0)$ (resp. $\Gamma>\Gamma(0)$ ) sufficiently small and fixed $p$, there exists $M$ such that $\Gamma$ is the minimal (resp. maximal) gap for $S_{p, M}(\Omega)$. Let $V^{0}$ be a potential in $L^{p}(\Omega)$ such that $\Gamma\left(V^{0}\right)=\Gamma$, and define

$$
M(\Gamma):=\left\{V \in L^{p}(\Omega): \Gamma(V)=\Gamma\right\} .
$$

Finally, define the functional $\rho: M(\Gamma) \rightarrow \mathscr{R}^{+}$by $\rho(V):=\|V\|_{p}^{p}$. 
Proposition II.2. The functional $\rho$ attains its minimum on $M(\Gamma)$ at some $V^{*}$, which is simultaneously an optimizer for $\Gamma(V)$ on $S_{p, M}$.

Proof. The argument used here is modeled on one in [18].

Let $\left\{V^{(k)}\right\}$ be a minimizing sequence for $\rho(V)$ of elements of $M(\Gamma)$. Then it is bounded in $L^{p}$ norm and we can extract a weakly convergent subsequence with limit $V^{*},\left\|V^{*}\right\|_{p} \leq M$. As in the proof of Theorem II.1, we have $\lim _{k} \Gamma\left(V^{(k)}\right)=\Gamma\left(V^{*}\right)$. We shall show later that in fact $\left\|V^{*}\right\|_{p}=M$.

The next task is to characterize the optimal potentials in $S_{p, M}$. We shall denote the gap minimizer $\left\langle V^{\sharp}, \psi_{j}^{\sharp}, E_{j}^{\sharp}\right\rangle$ as above, since in molecular physics a small gap corresponds to a "sharp" spectral line. In distinction to this we use $V^{b}$ for the gap maximizer, and when we do not distinguish between the optimizers it seems natural to use $V^{\natural}$. The basic idea is to subject $V^{\natural}$ to generic perturbations and to use the formula for the first-order change induced in $\Gamma$ to find conditions on $V^{\natural}$. Some of the ideas we use were developed by us in [2], [3], [8], [9], [11], [24].

Definition. A real-valued, bounded, measurable function $P(x)$ on $\Omega$ is an admissible perturbation of $V$ iff $\operatorname{dist}\left(V+\kappa P, S_{p, M}\right)=o(\kappa)$. It is strongly admissible iff $V+\kappa P \in S_{p, M}$ for all sufficiently small $\kappa$. An admissible perturbation is thus either strongly admissible or tangential to $\partial S_{p, M}$. On occasion we will refer to perturbations that are admissible only for positive or only for negative $\kappa$, but in the absence of an explicit restriction, $\kappa$ may have either sign.

We recall that if $E_{j}(V)$ is nondegenerate, then

$$
\frac{d E_{j}(V+\kappa P)}{d \kappa}=\int_{\Omega} P(x) \psi_{j}^{2} d x \quad \text { at } \kappa=0
$$

[5], [12], [16], [21] (in fact this is true for all $P \in L^{p}$, for the values of $p$ we consider). Therefore

$$
\frac{d \Gamma(V+\kappa P)}{d \kappa}=\int_{\Omega} P(x)\left(\psi_{2}^{2}-\psi_{1}^{2}\right) d x \quad(\kappa=0) .
$$

A complication arises when $E_{j}$ is degenerate [5], [12], [16]: $E_{j}$ can split into a cluster of eigenvalues $E_{j, m}$, which can be considered as a set of differentiable functions near $\kappa=0$, but those functions do not ordinarily correspond to the ordering of eigenvalues given by the minmax principle. For example, the lowest one for $\kappa<0$ will typically 
be the highest for $\kappa>0$. The ground-state is nondegenerate, but if $E_{2}$ is degenerate, then (2.5) is replaced by

$$
\frac{d \Gamma_{m}(V+\kappa P)}{d \kappa}=\int_{\Omega} P(x)\left(\psi_{2, m}^{2}-\psi_{1}^{2}\right) d x \quad(\kappa=0),
$$

where $\Gamma_{m}=E_{2, m}-E_{1}$, and the orthonormal eigenfunctions $\psi_{2, m}$ are specially chosen so that

$$
\int_{\Omega} \psi_{2, j} P \psi_{2, m} d x=0 \text { for } j \neq m
$$

We remark that in cases of highly symmetric $\Omega$, we believe that $E_{2}$ is indeed degenerate at maximum in a nontrivial way (symmetry is broken). For the minimizer, however, this does not happen.

We now state our main theorems. We start with the case $p=\infty$, and optimize over the set $S_{\infty, M}^{\prime}=\{V: 0 \leq V(x) \leq M\}$. This is essentially the same as $S_{\infty, M / 2}$, but is more convenient.

Note. In the absence of an explicit statement to the contrary, all set theoretic or topological operations occurring below should be understood to be carried out relative to $\Omega$, except for those referring to $\Omega$ itself and the operation of finding the support of a function (both of which should be taken relative to $\mathscr{R}^{n}$ ). By the support of an $L^{p}$ function $V$ we shall mean its distributional support. For a set $X, X^{c}$ will denote the complement, $\bar{X}$ the closure, and $\partial X$ the boundary.

TheOREM II.3. Let $\Omega$ and $H$ be as above, with $V$ constrained to $S_{\infty, M}^{\prime}$. Then

(a) $E_{2}\left(V^{\sharp}\right)$ is nondegenerate.

(b) Let $B^{\sharp}$ denote the support of $V^{\sharp}$. Then

$$
V^{\sharp}=M \chi_{B^{\sharp}}(x) \text { a.e. }
$$

Moreover,

$$
\psi_{1}^{\sharp}(x) \geq\left|\psi_{2}^{\sharp}(x)\right| \quad \text { on } B^{\sharp} \quad \text { and } \quad \psi_{1}^{\sharp}(x)<\left|\psi_{2}^{\sharp}(x)\right| \quad \text { on }\left(B^{\sharp}\right)^{c},
$$

and therefore $B^{\sharp}=\left\{x: \psi_{1}^{\sharp}(x) \geq\left|\psi_{2}^{\sharp}(x)\right|\right\}$.

(c) $B^{\sharp}$ is connected. 
THEOREM II.4. Let $\Omega$ and $H$ be as above, with $V$ constrained to $S_{\infty, M}^{\prime}$. Then either $E_{2}\left(V^{b}\right)$ is degenerate, or else in place of $(\mathrm{b})$ and (c) of the foregoing theorem we have:

$\left(b^{\prime}\right)$ Let the set $B^{b}$ denote the support of $V^{b}$. Then

$$
V^{b}=M \chi_{B^{b}}(x) \text { a.e. }
$$

Moreover,

(2.9) $\psi_{1}^{b}(x) \leq\left|\psi_{2}^{b}(x)\right| \quad$ on $B^{b}$ (with equality holding only on $\partial B^{b}$ ) and $\psi_{1}^{b}(x) \geq\left|\psi_{2}^{b}(x)\right|$ on $\left(B^{b}\right)^{c}$. Therefore,

$$
\left(B^{b}\right)^{\text {int }}=\left\{x: \psi_{1}^{b}(x)<\left|\psi_{2}^{b}(x)\right|\right\} .
$$

$\left(c^{\prime}\right)\left(B^{b}\right)^{c}$ is connected.

Theorem II.5. Let $\Omega$ and $H$ be as above, with $V$ constrained to $S_{p, M}, n / 2<p<\infty$. Then

(a) $E_{2}\left(V^{\sharp}\right)$ is nondegenerate; and

(b) $\operatorname{supp}\left(V^{\sharp}\right)=\bar{\Omega}$, and $V^{\sharp}$ and $\psi_{j}^{\sharp}$ are related by

$$
\psi_{2}^{\sharp 2}-\psi_{1}^{\sharp 2}=-c\left|V^{\sharp}\right|^{p-2} V^{\sharp}
$$

for some constant $c>0$. In particular, $\left|\psi_{2}^{\sharp}\right| \leq \psi_{1}^{\sharp}$ on $B_{+}^{\sharp}:=\operatorname{supp}\left(V_{+}^{\sharp}\right)$ and $\left|\psi_{2}^{\sharp}\right| \geq \psi_{1}^{\sharp}$ on $B_{-}^{\sharp}:=\operatorname{supp}\left(V_{-}^{\sharp}\right)$, where $V_{+}^{\sharp}:=\max \left(V^{\sharp}, 0\right)$ and $V_{-}^{\sharp}:=\min \left(V^{\sharp}, 0\right)$. In addition, $\left|\psi_{2}^{\sharp}\right|$ cannot equal $\psi_{1}^{\sharp}$ on the interior of $B_{-}^{\sharp}$.

(c) $B_{+}^{\sharp}$ is connected.

(d) $V^{\sharp} \in C(\Omega)$, and

$$
\int_{\Omega}\left|V^{\sharp}\right|^{p-2} V^{\sharp} d x=0 .
$$

Theorem II.6. Let $\Omega$ and $H$ be as above, with $V$ constrained to $S_{p, M}, n / 2<p<\infty$. Then either $E_{2}\left(V^{b}\right)$ is degenerate, or else in place of (b) and (c) of the foregoing theorem we have

$\left(\mathrm{b}^{\prime}\right) \operatorname{supp}\left(V^{\mathrm{b}}\right)=\bar{\Omega}$, and $V^{\mathrm{b}}$ and $\psi_{j}^{\mathrm{b}}$ are related by

$$
\psi_{2}^{b 2}-\psi_{1}^{b 2}=+c\left|V^{b}\right|^{p-2} V^{b}
$$

for some constant $c>0$. In particular, $\left|\psi_{2}^{b}\right| \geq \psi_{1}^{b}$ on $B_{+}^{b}:=\operatorname{supp}\left(V_{+}^{b}\right)$ and $\left|\psi_{2}^{b}\right| \leq \psi_{1}^{b}$ on $B^{b}:=\operatorname{supp}\left(V_{-}^{b}\right)$, where $V_{+}^{b}:=\max \left(V^{b}, 0\right)$ and 
$V_{-}^{b}:=\min \left(V^{b}, 0\right)$. In addition, $\left|\psi_{2}^{b}\right|$ cannot equal $\psi_{1}^{b}$ on the interior of $B_{+}^{b}$.

$\left(\mathrm{c}^{\prime}\right) \Omega \backslash B_{+}^{b}$ is connected.

$\left(\mathrm{d}^{\prime}\right) V^{b} \in C(\Omega)$, and

$$
\int_{\Omega}\left|V^{b}\right|^{p-2} V^{b} d x=0
$$

Properties (b) and $\left(\mathbf{b}^{\prime}\right)$ are the switching principles referred to in the introduction. They state that the potential switches on and off whenever the sign of $\left|\psi_{2}^{\sharp}\right|-\psi_{1}^{\sharp}$ changes. The physical interpretation of properties (c) is that the smallest possible gaps are caused by tunneling through single barriers, as in double-well problems. The proofs for minimizers will be done in several steps. The proofs for maximizers are almost exactly the same except for some sign changes, and the need to assume nondegeneracy. For simplicity of notation we drop the $\sharp$ throughout.

\section{Proof.}

Step 1. Theorem II.3, (b), first part: We first assume that $E_{2}$ is nondegenerate, and show that $\psi_{2}^{2}(x)=\psi_{1}^{2}(x)$ on the set $T:=\{x:$ $0<V(x)<M\}$, which therefore has measure 0 :

Write $T=\bigcup_{k=1}^{\infty} T_{k}$, where

$$
T_{k}=\left\{x: \frac{1}{k}<V(x)<M-\frac{1}{k}\right\},
$$

and assume that at least one of these is of positive measure. For any $x_{0} \in T_{k}$ and any measurable sequence of subsets $G_{k, j} \subset T_{k}$ containing $x_{0}$, perturbations of the form $P=\chi_{G_{k, j}}$ are admissible, so from (2.5),

$$
0=\frac{d \Gamma(V+\kappa P)}{d \kappa}=\int_{G_{k, j}}\left(\psi_{2}^{2}-\psi_{1}^{2}\right) d x \quad(\kappa=0) .
$$

Dividing by $\mu\left(G_{k, j}\right)$ and letting $G_{k, j}$ shrink to $x_{0}$ as $j \rightarrow \infty$, we find by the Lebesgue Density Theorem [22, p. 158] that

$$
\psi_{2}^{2}(x)=\psi_{1}^{2}(x) \text { on } T_{k} \text { and therefore on } T \text {. }
$$

We now claim that this can only occur on a set of measure zero. Let us assume to the contrary that $T_{+}:=\left\{x \in T: \psi_{2}(x)>0\right\}$ is of positive measure. (If the set where $\psi_{2}(x)<0$ were of positive measure, we could multiply $\psi_{2}$ by -1 .) Clearly $T=T_{+} \cup T_{-}$where $T_{-}:=\{x \in$ $\left.T: \psi_{2}(x)<0\right\}$, since $\psi_{2}^{2}=\psi_{1}^{2}$ on $T$ and $\psi_{1}$ does not vanish on $\Omega$. 
For a.e. $x \in T_{+}$there are sequences of points in $T_{+}$converging to $x$ from $n$ linearly independent directions, and we can differentiate a.e. by taking sequences of difference quotients evaluated at those points. This shows that $-\Delta \psi_{2}=-\Delta \psi_{1}$ a.e. in $T_{+}$. Substituting into the eigenvalue equation $(2.1)$, we find that $\left(E_{2}-E_{1}\right) \psi_{1}=0$ a.e. on $T_{+}$, which is impossible since $\psi_{1}(x)>0$ on $\Omega$.

Therefore, if $B$ denotes the distributional support of $V$, then $V=$ $M \chi_{B}(x)$ a.e.

Step 2. Theorem II.3, (b), remaining part, assuming nondegeneracy: To see that $\psi_{1}(x) \geq\left|\psi_{2}(x)\right|$ on $B$, observe that if $x \in B$ and $\left\{G_{j}\right\}$ is a sequence of sets containing $x$, then $P=\chi_{G_{j}}$ is an admissible perturbation for $\kappa \leq 0$. Therefore, from (2.5),

$$
0 \geq \frac{d \Gamma(V+\kappa P)}{d \kappa}=\int_{G_{j}}\left(\psi_{2}^{2}-\psi_{1}^{2}\right) d x \quad(\kappa=0) .
$$

Once again dividing by $\mu\left(G_{j}\right)$ and choosing $G_{j}$ to shrink nicely to $x$ as $j \rightarrow \infty$, it follows from the Lebesgue Density Theorem that $\psi_{1}^{2}(x) \geq \psi_{2}^{2}(x)$ on $B$.

A similar argument shows that $\psi_{1}^{2}(x) \leq \psi_{2}^{2}(x)$ on $B^{c}$. Moreover, the inequality on $B^{c}$ must be strict: Suppose that $\psi_{1}^{2}\left(x_{0}\right)=\psi_{2}^{2}\left(x_{0}\right)$ for some $x_{0} \in B^{c}$, and consider a ball $\mathscr{B}$ contained in $B^{c}$ and centered at $x_{0}$. We can assume without loss of generality that $\psi_{2}>0$ on $\mathscr{B}$ and we pick a potential $V$ of the equivalence class of a minimizing potential that is identically zero on $B^{c}$. The function $\Phi:=\psi_{1}-\psi_{2} \leq 0$ on $\mathscr{B}$ and attains its maximum at $x_{0}$. But $\Phi$ is subharmonic, since $\Delta \Phi=E_{2} \psi_{2}-E_{1} \psi_{1}>0$ on $\mathscr{B}$ and therefore $\psi_{2}=\psi_{1}$ on $\mathscr{B}$ by the maximum principle. This, however, is impossible as for (2.14).

REMARK. For a maximizing potential we can rule out the possibility of sets of measure zero on which $\psi_{1}(x)=\left|\psi_{2}(x)\right|$ in the interior of the support of $V$. Indeed, pick a $V$ from the equivalence class of a maximizing potential, such that $V$ is identically $M$ on $B$. We know that $\psi_{1}(x) \leq\left|\psi_{2}(x)\right|$ on $B$. If for some $x_{0} \in B^{\text {int }}, \psi_{1}\left(x_{0}\right)=\left|\psi_{2}\left(x_{0}\right)\right|$ and $\mathscr{B}$ is a ball in $B^{\text {int }}$ centered at $x_{0}$, we conclude (by assuming that $\psi_{2}>0$ in $\left.\mathscr{B}\right)$ that the function $\Phi:=\psi_{1}-\psi_{2}$ has a nonnegative maximum, viz., 0 , at $x_{0}$. Yet in $\mathscr{B} \Phi$ satisfies

$$
\Delta \Phi-M \Phi=E_{2} \psi_{2}-E_{1} \psi_{1}>0
$$


By the maximum principle [7, Chapter 3], we conclude that $\Phi$ must vanish identically on $B^{\text {int }}$, and thus that $\psi_{1}(x)=\psi_{2}(x)$ on a set of positive measure. This is a contradiction.

Step 3. Theorem II.3, (a): We now show that $E_{2}$ cannot be degenerate. If $E_{2}$ were $r$-fold degenerate, then for any particular strongly admissible perturbation $P(x)$, the cluster of eigenvalues $\left\{E_{2, m}(\kappa)\right\}$ into which $E_{2}$ would split could be arranged to be analytic in $\kappa$ at $\kappa=0$, and likewise for the associated orthonormalized eigenfunctions $\left\{\psi_{2}, m\right\}$ (depending on $P$ ). If

$$
\frac{d \Gamma_{m}}{d \kappa}<0 \quad(\kappa=0)
$$

for any $m$, then we would have $\Gamma\left(\kappa_{0}\right) \leq \Gamma_{m}\left(\kappa_{0}\right)<\Gamma(0)$ for some $\kappa_{0}>0$, which is impossible since $\Gamma(0)$ is a minimum. There is likewise a contradiction if

$$
\frac{d \Gamma_{m}}{d \kappa}>0 \quad(\kappa=0)
$$

for any $m$, because then we could find a $\kappa_{0}<0$ for which $\Gamma\left(\kappa_{0}\right) \leq$ $\Gamma_{m}\left(\kappa_{0}\right)<\Gamma(0)$. Therefore

$$
\frac{d \Gamma_{m}}{d \kappa}=\int_{\Omega} P(x)\left(\psi_{2, m}^{2}-\psi_{1}^{2}\right) d x=0 \quad(\kappa=0)
$$

for all admissible perturbations and all $m$. Suppose now $\psi$ is any normalized vector in the eigenspace for $E_{2}$, so that

$$
\psi=\sum_{j=1}^{r} c_{j} \psi_{2, j}, \quad \sum_{j=1}^{r}\left|c_{j}\right|^{2}=1 .
$$

Because of (2.7),

$$
\begin{aligned}
\int_{\Omega} P(x)\left(\psi^{2}-\psi_{1}^{2}\right) d x & =\int_{\Omega} P(x)\left(\sum_{j=1}^{r}\left|c_{j}\right|^{2} \psi_{2, j}^{2}-\psi_{1}^{2}\right) d x_{2} . \\
& =\sum_{j=1}^{r}\left|c_{j}\right|^{2} \int_{\Omega} P(x)\left(\psi_{2 j}^{2}-\psi_{1}^{2}\right) d x \\
& =0
\end{aligned}
$$

We may now argue as in Steps 1 and 2, restricting first to sets $T_{k}$ to conclude that for some set $B, V(x)=M \chi_{B}(x)$ a.e. We shall now argue that on $B$,

$$
\psi_{1}^{2}(x) \geq \psi^{2}(x)
$$


as before, and that this holds for each and every normalized vector $\psi$ in the eigenspace of $E_{2}$ : If $P$ is a positive perturbation supported in $B^{c}$ (resp. $\left.B\right)$, then as before we must have

$$
\frac{d \Gamma_{m}}{d \kappa} \geq 0 \quad(\text { resp. } \leq 0)
$$

when $\kappa=0$. As in (2.15) we find

$$
\int_{\Omega} P(x)\left(\psi^{2}-\psi_{1}^{2}\right) d x \geq 0 \quad(\text { resp. } \leq 0),
$$

and (2.16) is established a.e. by the Lebesgue Density Theorem.

Suppose, finally, that there are two orthonormal vectors, $\psi_{2, a}$ and $\psi_{2, b}$ in the second eigenspace, and that $x_{0}$ is a point on $\partial B \cap \Omega$, so that we may take $\psi_{2, a}\left(x_{0}\right)=\psi_{2, b}\left(x_{0}\right)=\psi_{1}\left(x_{0}\right) \neq 0$. Then the vector

$$
\psi(x)=\frac{1}{\sqrt{2}}\left(\psi_{2, a}(x)-\psi_{2, b}(x)\right)=0 \quad \text { when } x=x_{0} .
$$

It would follow that $\psi_{1}^{2}(x)>\psi^{2}(x)$ on part of $\Omega \backslash B$, contradicting (2.16).

We now turn to the cases where $n / 2<p<\infty$.

Step 4. Theorem II.5, (b), assuming nondegeneracy: It is clear that the minimizing potential satisfies $\|V\|_{p}=M$, as otherwise every bounded perturbation is admissible, and we would find as in Step 1 that $\psi_{2}^{2}(x)=\psi_{1}^{2}(x)$ throughout $\Omega$, which is impossible. In addition, any bounded, measurable perturbation such that

$$
\operatorname{supp}(P) \subset \Omega \backslash \operatorname{supp}(V)
$$

is admissible, so the same argument implies that $\operatorname{supp}(V)=\bar{\Omega}$.

Perturbations admissible for both positive and negative $\kappa$ are tangential to $\partial S_{p, M}$ in $L^{p}$ in the sense that

$$
\int_{\Omega}|V(x)|^{p-2} V(x) P(x) d x=0,
$$

according to an easy calculation. According to (2.5), at optimum,

$$
\int_{\Omega} P(x)\left(\psi_{2}^{2}-\psi_{1}^{2}\right) d x=0
$$

for all bounded, measurable $P$ satisfying (2.18). It is not difficult to see that this implies the proportionality (2.10) for some constant (cf. [3, p. 1773]). Actually, for an arbitrary minimizing potential this 
proportionality need only hold a.e. Nonetheless, since $\psi_{1}^{\sharp}$ and $\psi_{2}^{\sharp}$ are both continuous and changes of $V^{\sharp}$ on sets of measure 0 do not affect anything, we shall always choose the gap-minimizing potential as the distinguished member of its equivalence class such that the proportionality $(2.10)$ holds everywhere. It is this assumption that allows us to assert in (d) that $V^{\sharp} \in C(\Omega)$. Similar comments apply to $V^{b}$ in Theorem II.6. To see that the proportionality constant $-c$ is negative, note that $P(x)=\chi_{T}(x)$ is admissible for any measurable set $T \subset \operatorname{supp}\left(V_{+}\right)$and negative $\kappa$, or for $T \subset \operatorname{supp}\left(V_{-}\right)$and positive $\kappa$. In the former case $\Gamma \leq 0$ and in the latter $\Gamma \geq 0$. We now argue as in Step 2 to conclude the switching principle and concluding statement of Property (b). The fact that $\left|\psi_{2}\right| \neq \psi_{1}$ on $\operatorname{int}\left(B_{-}^{\sharp}\right)$ is proved as in Step 2.

Step 5. Theorem II.5, (a): To establish nondegeneracy in this case, we once again consider the cluster of eigenvalues $\left\{E_{2, m}(\kappa)\right\}$ into which $E_{2}$ splits under perturbation. Let $\left\{\psi_{2}, m(\kappa)\right\}$ denote the associated orthonormalized eigenfunctions. Since $E_{2, m}(\kappa)$ are chosen to be analytic in a neighborhood of $\kappa=0$, we argue as in Steps 3 and 4 to conclude that on $\Omega$

$$
\psi^{2}-\psi_{1}^{2}=-c|V|^{p-2} V
$$

for every normalized $\psi$ in the eigenspace of $E_{2}$ and some constant $c>0$, which may in general depend on $\psi$. In particular, any such $\psi$ satisfies the switching principle, and we can obtain a contradiction as in Step 3.

Step 6. Theorem II.5, (d): The regularity claim for $V$ follows from the regularity of $\psi_{1}$ and $\psi_{2}$ and the algebraic relationship (2.10) of part (b), which holds everywhere on $\Omega$ by virtue of our convention for the choice of $V^{\sharp}$ discussed in Step 4 above. The integral identity results from integrating $(2.10)$ and recalling that $\psi_{1}$ and $\psi_{2}$ are normalized.

Step 7. Theorems II.3 and II.5, (c) ("Gap-minimizing potentials have no islands"): For this proof we denote both $B^{\sharp}$ and $B_{+}^{\sharp}$ by $B$. Clearly the nodal set $\left\{x: \psi_{2}(x)=0\right\}$ belongs to a connected component of $B$. The nodal set separates $\Omega$ into two nodal domains, so suppose that $B$ were to contain two disjoint regions $\Omega_{1}$ and $\Omega_{2}$, one of which, say $\Omega_{1}$, includes the nodal set, while $\Omega_{2}$ lies within one of the nodal domains. Without loss of generality, we assume that 
$\psi_{2}>0$ on $\Omega_{2}$. We have established that $\psi_{1}>\psi_{2}$ a.e. on $\Omega_{2}$ and $\psi_{1}=\psi_{2}$ on $\partial \Omega_{2}$. We use $u(x):=\psi_{1}(x)-\psi_{2}(x)$ as a trial function for the Dirichlet eigenvalue problem for $H$ restricted to $\Omega_{2}$, noting that the lowest eigenvalue of this restriction lies above $E_{2}$ because $\Omega_{2}$ lies within a nodal domain. Hence the Rayleigh-Ritz inequality would imply that

$$
\begin{aligned}
& E_{2} \int_{\Omega_{2}}\left(\psi_{1}-\psi_{2}\right)^{2} d x \leq \int_{\Omega_{2}}\left(\psi_{1}-\psi_{2}\right) H\left(\psi_{1}-\psi_{2}\right) d x \\
& \quad=\int_{\Omega_{2}}\left(\psi_{1}-\psi_{2}\right)\left(E_{1} \psi_{1}-E_{2} \psi_{2}\right) d x \\
& \quad=E_{1} \int_{\Omega_{2}}\left(\psi_{1}-\psi_{2}\right)^{2} d x-\left(E_{2}-E_{1}\right) \int_{\Omega_{2}}\left(\psi_{1}-\psi_{2}\right) \psi_{2} d x \\
& \quad<E_{1} \int_{\Omega_{2}}\left(\psi_{1}-\psi_{2}\right)^{2} d x
\end{aligned}
$$

which would contradict $E_{2}>E_{1}$.

III. Optimal potentials in one dimension. In this section $\Omega=(0,1)$ and $H=-d^{2} / d x^{2}+V$ acts on the subspace of $L^{2}(0,1)$ corresponding to Dirichlet boundary conditions. We consider $V \in S_{p, M}(\Omega)$ initially for $1<p<\infty$, leaving the case $p=\infty$ for later. There are several properties special to one dimension that make our problem easier to handle and allow us to push the results in $\S I I$ further. One useful fact is that all eigenvalues of $H$ are nondegenerate, so the results of the previous section stated for maximizers will always hold.

Let us begin with a discussion of $V^{\sharp}$, a gap minimizer for $S_{p, M}(\Omega)$. Let $\psi_{1}^{\sharp}$ and $\psi_{2}^{\sharp}$ denote the corresponding ground state and the first excited state. It is not hard to see from the proof of Step 7 that in one dimension $\psi_{1}^{\sharp}(x)=\left|\psi_{2}^{\sharp}(x)\right|$ at most at two points in $(0,1)$ (see also [9]), so the positive support of the minimizing potential is an interval $[a, b]$ for $0 \leq a<b \leq 1$. The minimizer is a double-well potential provided that $a>0$ and $b<1$. We shall show that this is indeed the case.

We start by recalling the relation (2.10):

$$
\psi_{2}^{\sharp 2}-\psi_{1}^{\sharp 2}=-c\left|V^{\sharp}\right|^{p-2} V^{\sharp},
$$

which holds throughout $[0,1]$. Since $\psi_{1,2}$ are continuous, so is $V^{\sharp}$, and in fact by elliptic regularity we conclude that it is infinitely differentiable in the interior of $\operatorname{supp}\left(V_{+}^{\sharp}\right)$ and $\operatorname{supp}\left(V_{-}^{\sharp}\right)$. (It is easy to see that for $p=1+1 / m, m=1,2, \ldots, V^{\sharp} \in C^{\infty}(0,1)$, but otherwise 
there may be nonsmoothness at the points where $V=0$.) Differentiating both sides of $(2.10)$ on $\operatorname{supp}\left(V_{+}^{\sharp}\right)$ and $\operatorname{supp}\left(V_{-}^{\sharp}\right)$ and substituting from the Schrödinger equation we obtain a non-linear differential equation connecting $V^{\sharp}$ with its wave functions $\psi_{j}^{\sharp}, j=1,2$.

THEOREM III.1. Let $V^{\sharp}$ minimize the fundamental gap in $S_{p, M}(\Omega)$ for fixed $M \geq 0$ and $1<p<\infty$. Then

$$
\text { 1) }-\frac{d^{2}}{d x^{2}}\left(c\left|V^{\sharp}\right|^{p-2} V^{\sharp}\right)+\left(4-\frac{2}{p}\right) c\left|V^{\sharp}\right|^{p}=4\left(E_{1}^{\sharp} \psi_{1}^{\sharp 2}-E_{2}^{\sharp} \psi_{2}^{\sharp 2}\right)+C,
$$

where

$$
\begin{aligned}
C & =-\left.\frac{d^{2}}{d x^{2}}\left(c\left|V^{\sharp}\right|^{p-2} V^{\sharp}\right)\right|_{x=0}=-\left.\frac{d^{2}}{d x^{2}}\left(c\left|V^{\sharp}\right|^{p-2} V^{\sharp}\right)\right|_{x=1} \\
& =2\left(\left(\frac{d \psi_{2}^{\sharp}(0)}{d x}\right)^{2}-\left(\frac{d \psi_{1}^{\sharp}(0)}{d x}\right)\right)^{2} \\
& =2\left(\left(\frac{d \psi_{2}^{\sharp}(1)}{d x}\right)^{2}-\left(\frac{d \psi_{1}^{\sharp}(1)}{d x}\right)^{2}\right) .
\end{aligned}
$$

Proof. Once again we drop the $\sharp$ for convenience. On $\operatorname{supp}\left(V_{+}\right)$, (2.10) becomes

$$
\psi_{2}^{2}-\psi_{1}^{2}=-c V^{p-1} .
$$

Differentiating both sides in the interior of this set we get:

$$
\begin{gathered}
2 \psi_{2}^{\prime} \psi_{2}-2 \psi_{1}^{\prime} \psi_{1}=-\frac{d}{d x}\left(c V^{p-1}\right), \\
2 \psi_{2}^{\prime 2}-2 \psi_{1}^{\prime 2}+2 \psi_{2} \psi_{2}^{\prime \prime}-2 \psi_{1} \psi_{1}^{\prime \prime}=-\frac{d^{2}}{d x^{2}}\left(c V^{p-1}\right) .
\end{gathered}
$$

Now we substitute for $\psi_{1,2}^{\prime \prime}$ from the Schrödinger equation (2.1) and use (3.3). This yields

$$
2\left(\psi_{2}^{\prime 2}-\psi_{1}^{\prime 2}\right)-2 V\left[c V^{p-1}\right]+2\left(E_{1} \psi_{1}^{2}-E_{2} \psi_{2}^{2}\right)=-\frac{d^{2}}{d x^{2}}\left(c V^{p-1}\right) .
$$

Differentiating again,

$$
\begin{gathered}
4\left(\psi_{2}^{\prime} \psi_{2}^{\prime \prime}-\psi_{1}^{\prime} \psi_{1}^{\prime \prime}\right)-2 V \frac{d}{d x}\left[c V^{p-1}\right]-2 V^{\prime}\left[c V^{p-1}\right] \\
+4\left(E_{1} \psi_{1} \psi_{1}^{\prime}-E_{2} \psi_{2} \psi_{2}^{\prime}\right)=-\frac{d^{3}}{d x^{3}}\left[c V^{p-2}\right] .
\end{gathered}
$$


Once again we substitute for $\psi_{j}^{\prime \prime}$ and this time use (3.4). The result is

$-4 V \frac{d}{d x}\left[c V^{p-1}\right]-2 V^{\prime}\left[c V^{p-1}\right]+8\left(E_{1} \psi_{1} \psi_{1}^{\prime}-E_{2} \psi_{2} \psi_{2}^{\prime}\right)=-\frac{d^{3}}{d x^{3}}\left[c V^{p-1}\right]$.

The first two terms on the left can be rewritten:

$$
-4 V \frac{d}{d x}\left[c V^{p-1}\right]-2 V^{\prime}\left[c V^{p-1}\right]=-\left(4-\frac{2}{p}\right) \frac{d}{d x}\left[c V^{p}\right] .
$$

Integrating (3.6) then yields

$$
-\left(4-\frac{2}{p}\right)\left[c V^{p}\right]+4\left(E_{1} \psi_{1}^{2}-E_{2} \psi_{2}^{2}\right)+C=-\frac{d^{2}}{d x^{2}}\left[c V^{p-1}\right]
$$

where $C$ is the constant of integration.

Similarly, on the interior of $\operatorname{supp}\left(V_{-}\right)$we find that

$$
-\left(4-\frac{2}{p}\right)\left[c(-V)^{p}\right]+4\left(E_{1} \psi_{1}^{2}-E_{2} \psi_{2}^{2}\right)+C=-\frac{d^{2}}{d x^{2}}\left[c(-V)^{p-1}\right] .
$$

That the constant of integration $C$ is the same throughout $[0,1]$ follows from the continuity of $V$ and $\psi_{1,2}$ at both $a$ and $b$. The equations can therefore be combined, yielding equation (3.1) on the whole interval.

REMARKS. 1. An interesting special case is $p=2$, for which (3.1) becomes

$$
-c V^{\prime \prime}+3 c V^{2}=4\left(E_{1} \psi_{1}^{2}-E_{2} \psi_{2}^{2}\right)+C .
$$

2. Obviously we can use equation $(2.10)$ to eliminate either $\psi_{1}$ or $\psi_{2}$ from (3.1).

3. A gap maximizer $V^{b}$ for $S_{p, M}(\Omega)$ is connected with its eigenfunctions $\psi_{j}(j=1,2)$ via a similar non-linear differential equation.

Corollary III.2. Let $V^{\sharp}$ be a gap minimizer for $S_{p, M}(\Omega), 1<$ $p<\infty$. Then

$$
\operatorname{supp}\left(V_{-}\right)=[0, a] \cup[b, 1] \text { and } \operatorname{supp}\left(V_{+}\right)=[a, b]
$$

for some $0<a<b<1$. In other words, $V^{\sharp}$ consists of two wells separated by a barrier.

Proof. Integrate both sides of (3.1) from 0 to 1:

$$
-\left.\frac{d}{d x}\left(c|V|^{p-2} V\right)\right|_{0} ^{1}+\left(4-\frac{2}{p}\right) c M^{p}=4\left(E_{1}-E_{2}\right)+C
$$


(as usual we drop the $\sharp$ ). Therefore, since

$$
-\left.\frac{d}{d x}\left(c|V|^{p-2} V\right)\right|_{0} ^{1}=\left.2\left(\psi_{2} \psi_{2}^{\prime}-\psi_{1} \psi_{1}^{\prime}\right)\right|_{0} ^{1}=0
$$

it follows that

$$
E_{2}-E_{1}=+\frac{1}{4} C-\left(1-\frac{1}{2 p}\right) c M^{p}
$$

Since $c>0$ and $E_{2}>E_{1}$, this shows that $C$ is strictly positive.

Suppose now $a=0$, i.e., $V<0$ on a single interval $(b, 1)$ and thus there is only one well. (The case $b=1$ is handled similarly.) Choose the sign of $\psi_{2}$ to be positive to the left of the node. Then $\psi_{1}(0)=\psi_{2}(0)=0$ and by Theorem II.5, $\psi_{1}(x)>\psi_{2}(x)>0$ for small $x>0$. Therefore, $\psi_{1}^{\prime}(0) \geq \psi_{2}^{\prime}(0)>0$, but that contradicts (3.2), since $C>0$.

A similar and somewhat more involved argument proves a related fact about gap maximizers.

Corollary III.3. Let $V^{b}$ be a gap maximizer in $S_{p, M}(\Omega), 1<$ $p<\infty$. Then for some $a, b$, with $0<a<b<1, \operatorname{supp}\left(V_{-}^{b}\right)=[a, b]$, and $\operatorname{supp}\left(V_{+}^{b}\right)=[0, a] \cup[b, 1]$.

In other words, $V^{b}$ consists of two barriers with a single well in between.

Proof. As usual, we drop the superscript ${ }^{b}$. Suppose that the claim were false. Then we may assume without loss of generality that $\operatorname{supp}\left(V_{+}\right)=[0, a]$ and $\operatorname{supp}\left(V_{-}\right)=[a, 1]$ (recall Theorem II.6 $\left(\mathbf{c}^{\prime}\right)$ ), and we take $\psi_{2}$ to be positive to the left of its node. From Theorem II. $6\left(\mathbf{b}^{\prime}\right), 0<\psi_{1}(x)<\psi_{2}(x)$ in a small right neighborhood of 0 (where both functions are increasing), while $0<-\psi_{2}(x)<\psi_{1}(x)$ on a small left neighborhood of 1 (where both functions are decreasing). Therefore, $\psi_{1}^{\prime}(0)^{2} \leq \psi_{2}^{\prime}(0)^{2}$ and $\psi_{1}^{\prime}(1)^{2} \geq \psi_{2}^{\prime}(1)^{2}$. This implies that

$$
C=2\left(\psi_{2}^{\prime}(0)^{2}-\psi_{1}^{\prime}(0)^{2}\right)=2\left(\psi_{2}^{\prime}(1)^{2}-\psi_{1}^{\prime}(1)^{2}\right)=0,
$$

i.e., $\psi_{1}^{\prime}(0)=\psi_{2}^{\prime}(0)$. Let $x_{0}$ denote the node of $\psi_{2}$, and consider the original equations on $\left[0, x_{0}\right]$ :

$$
-\psi_{j}^{\prime \prime}+\left(V(x)-E_{j}\right) \psi_{j}=0, \quad j=1,2,
$$

with $\psi_{1}(0)=\psi_{2}(0)=0$, and $\psi_{1}^{\prime}(0)=\psi_{2}^{\prime}(0)$. Observe that with 
our choice of sign, $\psi_{j}(x)>0$ on $\left(0, x_{0}\right)$. Let $W(x):=\psi_{1} \psi_{2}^{\prime}-$ $\psi_{2} \psi_{1}^{\prime}$. Then $W^{\prime}(x)=\left(E_{1}-E_{2}\right) \psi_{1} \psi_{2}<0$ on this open interval. Since $W(0)=0, W(x)<0$ on $\left(0, x_{0}\right]$.

Now define

$$
f(x)=\frac{\psi_{2}(x)}{\psi_{1}(x)} \text { for } 0<x \leq x_{0}, \quad \text { and } \quad f(0)=1 .
$$

Because of (3.9), $f \in C^{1}\left[0, x_{0}\right]$, and since

$$
f^{\prime}(x)=\frac{W(x)}{\psi_{1}^{2}(x)}<0,
$$

it follows that $f(x)<1$ on a right neighborhood of 0 . This, however, contradicts Theorem II.6.

We next draw on ideas of [18] to strengthen Proposition II. 2 in the one-dimensional situation:

Proposition III.4. The sets $M\left(\Gamma^{\natural}\right)$ and $\partial S_{p, M}(\Omega)$ have a common tangent space at $V^{\natural}$, i.e., for $P \in L^{p}(\Omega)$,

if and only if

$$
\int_{0}^{1}\left|V^{\natural}\right|^{p-2} V^{\natural} P d x=0
$$

$$
\int_{0}^{1} P\left(\psi_{2}^{\natural_{2}}-\psi_{1}^{\natural_{2}}\right) d x=0
$$

Proof. The special fact about one dimension is that the functional $\Gamma$ is Fréchet differentiable for any $1<p<\infty$, and for any $P \in L^{p}(\Omega)$,

$$
d_{V} \Gamma(P)=\int_{0}^{1} P(x)\left(\psi_{2}^{2}-\psi_{1}^{2}\right) d x
$$

where $\psi_{1}$ and $\psi_{2}$ are the ground state and first excited state associated with $V[\mathbf{1 8}]$. The functional $\rho$ defined in $\S I I$ (considered on all of $\left.L^{p}(\Omega)\right)$ is similarly Fréchet differentiable, and

$$
d_{V} \rho(P)=p \int_{0}^{1}|V|^{p-2} V P d x .
$$

The set $M\left(\Gamma^{\natural}\right)$ is a level surface of the functional $\Gamma$, while the set $\partial S_{p, M}(\Omega)$ is a level surface of $\rho$. By Proposition II.2, $V^{\natural}$ belongs to both of them. Moreover, both surfaces are real analytic submanifolds with tangent spaces at $V^{\text {b }}$ given by

(3.10) $\quad T_{V^{\natural}} M\left(\Gamma^{\natural}\right)=\operatorname{ker} d_{V^{\natural}} \Gamma^{\natural}$

$$
=\left\{P \in L^{p}: \int_{0}^{1} P(x)\left(\psi_{2}^{2}-\psi_{1}^{2}\right) d x=0\right\}
$$


and

$$
\text { (3.11) } T_{V^{\natural}} \partial S_{p, M}=\operatorname{ker} d_{V} \rho=\left\{P \in L^{p}: \int_{0}^{1}|V|^{p-2} V P d x=0\right\}
$$

[18, pp. 153-154] (our convention is to shift the tangent space to the origin in $L^{p}$ ).

That the functional $\rho$ is minimized (or maximized) at $V^{\natural}$ means that $\left.d_{V^{\natural}} \rho\right|_{T_{V^{\natural}} M\left(\Gamma^{\natural}\right)}$ vanishes. In other words, any $P$ satisfying (3.10) also satisfies (3.11), and thus belongs to $T_{V^{\sharp}} \partial S_{p, M}$. Similarly, since $\Gamma$ is minimized (or maximized) at $V^{\natural}$ we have $\left.d_{V^{\natural}} \Gamma\right|_{T_{V^{\natural}} \partial S_{p, M}\left(\Gamma^{\natural}\right)}=$ 0 . Thus any $P$ satisfying (3.11) also satisfies (3.10) and belongs to $T_{V^{\natural}} M\left(\Gamma^{\natural}\right)$.

For $p=2$ we can be more precise:

Proposition III.5. If $p=2$, then $V^{\natural}$ is an even function, i.e., $V^{\natural}(x)=V^{\natural}(1-x)$.

Proof. We continue to follow [18]. It is shown there (p. 44, Theorem 8) that for any eigenfunction $\psi_{m}^{\natural}$ of $V^{\natural}$,

$$
\int_{0}^{1} \frac{d}{d x} \psi_{m}^{\natural 2} \psi_{j}^{\natural 2} d x=0, \quad j=1,2 .
$$

Therefore (dropping the superscript),

$$
\int_{0}^{1} \frac{d}{d x} \psi_{m}^{2}\left(\psi_{2}^{2}-\psi_{1}^{2}\right)=0
$$

and by Proposition III.4, this implies that

$$
\int_{0}^{1} V \frac{d}{d x} \psi_{m}^{2} d x=0
$$

for all $m=1,2, \ldots$.

But this quantity can also be calculated as in [18, p. 74, Lemma 1], according to which

$$
\int_{0}^{1} V \frac{d}{d x} \psi_{m}^{2} d x=2 \frac{(-1)^{m}}{\dot{y}_{2}\left(1, E_{m}\right)} \sinh \left(\kappa_{m}\right),
$$

where $y_{2}\left(x, E_{m}\right)$ is a multiple of $\psi_{m}(x)$ normalized so that $y_{2}^{\prime}\left(0, E_{m}\right)$ $=1$. The final key fact is that $\kappa_{m}(V)=0$ for all $m$ if and only if $V$ is an even function [18, p. 62, Lemma 4], so the proposition is proved by comparing (3.12) and (3.13). 
We conjecture that this is true for all $p>1$. (The conjecture is settled affirmatively below for $p=\infty$ by entirely different methods.) For the remaining values of $p$, we can prove a weaker statement, namely:

Proposition III.6. Given a gap-optimizing potential $V^{\natural} \in S_{p, M}(\Omega)$ for $1<p<\infty$, there exists a continuous, even function $V^{\bullet} \in L^{2}(\Omega)$ such that

$$
\left\|V^{\bullet}\right\|_{2} \leq\left\|V^{\natural}\right\|_{2}, \quad\left\|V^{\bullet}\right\|_{p} \geq\left\|V^{\natural}\right\|_{p}, \quad \text { and } \quad \Gamma\left(V^{\bullet}\right)=\Gamma\left(V^{\natural}\right) .
$$

Proof. From (2.10) we know that $V^{\natural}$ is continuous and therefore belongs to $L^{2}(\Omega)$. We consider a submanifold $M(\Gamma)=\left\{V \in L^{2}(\Omega)\right.$ : $\left.\Gamma(V)=\Gamma\left(V^{\natural}\right)\right\}$ and the functional $\rho$ on $M(\Gamma)$ defined by $\rho(V):=$ $\|V\|_{2}^{2}$. As before, $\rho$ attains a minimum on $M(\Gamma)$, which we denote by $V^{\bullet}$, and we know that $V^{\bullet}$ is even. The first inequality follows. The second inequality holds because $V^{\natural}$ is an optimal potential in $S_{p, M}(\Omega)$.

Now we deal with the case $p=\infty$. The reason for having to consider it separately is that the functional $\rho(V)=\|V\|_{\infty}$ is not Fréchet differentiable. On the other hand, some things are easier here because gap minimizers have a particularly simple form, and were already discussed to some extent in [9]. We shall prove below that an optimal potential in $S_{\infty, M}^{\prime}$ is a symmetric square barrier of height $M$, by using the Sturm comparison theorem and Sturm Separation Theorem.

TheOREM III.7. Let $V^{\sharp}$ be a potential in $S_{\infty, M}^{\prime}$ minimizing the fundamental gap. Then $V^{\sharp}=M \chi_{B^{\sharp}}$, where

$$
B^{\sharp}=\left\{x:\left|\psi_{2}^{\sharp}(x)\right| \leq \psi_{1}^{\sharp}(x)\right\}=[a, 1-a] .
$$

Proof. We have already shown that $B$ is connected and $B=\{x$ : $\left.\left|\psi_{2}(x)\right| \leq \psi_{1}(x)\right\}$. What is left to show is that $B$ is symmetric.

Suppose that the barrier $B$ were not symmetric. For convenience we shift the interval from $[0,1]$ so that the node of $\psi_{2}$ is at 0 , and denote the shifted interval $\left[-c^{<}, c^{>}\right]$. Let $-a^{<}$denote the left end of the barrier and $a^{>}$the right end. We assume without loss of generality that $a^{<} \leq a^{>}$.

Write $U(x):=V\left(c^{>}-c^{<}-x\right)$ and $\phi_{2}(x):=\psi_{2}\left(c^{>}-c^{<}-x\right)$. Note that $\psi_{2}$ has a node at 0 , while $\phi_{2}$ has a node at $c^{>}-c^{<}$. Using the 
Sturm Comparison Theorem we conclude that

$$
c^{<}-a^{<}>c^{>}-a^{>}, \quad c^{<}<c^{>}, \text {and } a^{<}<a^{>} .
$$

There are now two possibilities to consider:

(1) $c^{>}-a^{>}=0$, i.e., the barrier stretches on the right all the way to the end of the interval; and

(2) $c^{>}-a^{>}>0$.

The first possibility will be eliminated by showing that such a potential cannot minimize the gap. Suppose that indeed $c^{>}-a^{>}=0$. Then we observe that:

(a) $E_{2}>M$ for $\psi_{2}$ must have a node somewhere in the interior of $B^{\sharp}$; and

(b) $E_{1}>M$ as well: Otherwise $\psi_{1}(x)=\sinh \left(\sqrt{M-E_{1}}\left(c^{>}-x\right)\right)$ in the barrier (up to a constant), and would thus be a decreasing function. Hence

$$
\psi_{1}\left(a^{<}\right)<\psi_{1}\left(-a^{<}\right)=\left|\psi_{2}\left(-a^{<}\right)\right|=\left|\psi_{2}\left(a^{<}\right)\right|,
$$

where the latter equality arises from the reflection symmetry of $\left|\psi_{2}\right|$ about 0 . Since $a^{<} \in B$, this leads to a contradiction.

(c) Therefore $\psi_{1}(x)$ must have a single maximum occurring at some point on $m$. By comparing Prüfer angles for $\psi_{1}(x)$ and its reflection $\varphi(x):=\psi_{1}\left(c^{>}-c^{<}-x\right)$ on the interval $\left[-c^{<}, c^{>}\right]$with both angles initialized to 0 at $x=-c^{<}$, we see that $m$ lies closer to $-c^{<}$than to $c^{>}$. It must also lie within the barrier, for otherwise $\psi_{1}(x)$ would be decreasing in $B$ and that would lead to the same contradiction as in (b). Finally, $m \geq 0$, since if $m<0$, then by symmetry

$$
\left|\psi_{2}\left(a^{<}\right)\right|=\left|\psi_{2}\left(-a^{<}\right)\right|=\psi_{1}\left(-a^{<}\right)=\psi_{1}\left(2 m+a^{<}\right)>\psi_{1}\left(a^{<}\right),
$$

which is impossible.

(d) Consider the interval $\left[-c^{<}, m\right]$. On this interval we can decrease $E_{2}$ if necessary until the node of $\psi_{2}$ coincides with $m$. Then we reflect symmetrically about $m$. This new problem has a smaller fundamental gap and a smaller interval length. If we scale the length back to 1 , we decrease the gap even further, while also decreasing the $\|V\|_{\infty}$. This produces a symmetric potential that has both a smaller $L^{\infty}$-norm and a smaller fundamental gap than the given potential. Therefore, a potential that stretches all the way to the end of the interval in one direction cannot minimize $\Gamma$.

The only remaining possibility is that $c^{>}-a^{>}>0$. In this case either $\psi_{1}\left(-a^{<}\right) \leq \psi_{1}\left(a^{>}\right)$or $\psi_{1}\left(-a^{<}\right)>\psi_{1}\left(a^{>}\right)$. Note that we can write down the wave functions $\psi_{j}(x), j=1,2$, explicitly. If 
$M-E_{j}<0$, then $\psi_{j}(x)$ is a trigonometric function (sin or cos) in the barrier, while if $M-E_{j}>0$, then $\psi_{j}(x)$ is a hyperbolic function (sinh or cosh), and, finally, if $E_{j}=M$, then $\psi_{j}$ is a linear function. Combining this with the fact that $\psi_{1}(x) \geq\left|\psi_{2}(x)\right|$ in the barrier, with equality only at $-a^{<}$and $a^{>}$, we notice that the second possibility, $\psi_{1}\left(-a^{<}\right)>\psi_{1}\left(a^{>}\right)$can occur only if $E_{2}>E_{1}>M$.

Now suppose $\psi_{1}\left(-a^{<}\right) \leq \psi_{1}\left(a^{>}\right)$. Let

$$
v_{1}(x):=\psi_{1}\left(x+a^{>}\right) \text {and } v_{2}(x):=\psi_{1}\left(-x-a^{<}\right) .
$$

Then

$$
\begin{gathered}
v_{1}(0)=\psi_{1}\left(a^{>}\right) \geq \psi_{1}\left(-a^{<}\right)=v_{2}(0) ; \\
v_{1}\left(-a^{<}-a^{>}\right)=\psi_{1}\left(-a^{<}\right) \leq \psi_{1}\left(a^{>}\right)=v_{2}\left(-a^{<}-a^{>}\right) ; \\
v_{1}\left(c^{>}-a^{>}\right)=0<v_{2}\left(c^{<}-a^{<}\right) .
\end{gathered}
$$

Therefore the function $w(x):=v_{1}(x)-v_{2}(x)$ solves the differential equation

$$
-u^{\prime \prime}+V u=E_{1} u
$$

on the interval $\left[-a^{<}-a^{>}, c^{>}-a^{>}\right]$and vanishes at least twice there (in fact, $w\left(-\left(a^{<}+a^{>}\right) / 2\right)=0$ and $w$ vanishes again on $\left[0, c^{>}-a^{>}\right]$). On the other hand, $v_{1}(x)$ is another solution of this equation in the same interval, linearly independent from $w(x)$ and nonvanishing in the interior. This contradicts the Sturm Separation Theorem. (The general fact here is that two independent solutions to a linear, homogeneous, second-order ordinary differential equation can cross at most once on any node-free interval.)

If the second possibility arises, i.e., $\psi_{1}\left(-a^{<}\right)>\psi_{1}\left(a^{>}\right)$, then, as already observed, $E_{2}>E_{1}>M$. The part of the proof above used to rule out $c^{>}-a^{>}=0$ can be repeated word for word to show that such a potential cannot be a gap minimizer. This finally covers all the cases and proves the theorem.

IV. Conclusions. The main goal of showing that optimally small gaps can be attributed to tunneling through barriers, as in doublewell problems, has been attained with varying degrees of analytical success, depending on the geometry and the nature of the constraints imposed, by Theorems II.3-II.6, III.1 and III.7, characterizing the gapoptimizing potentials. These theorems appear sufficiently definitive to allow numerical estimates of $\Gamma$ in many cases in one and two dimensions. The one-dimensional cases are straightforward, especially 
$S_{p, \infty}^{\prime}$, which is amenable to several numerical methods, including one that uses a dimension-independent algorithm: The basis of the algorithm is to regard the optimizers as fixed points of a mapping on $S_{p, M}$ or $S_{p, \infty}^{\prime}$ :

$$
V \rightarrow E_{1,2} \text { and } \psi_{1,2}
$$

followed by

$$
E_{1,2} \text { and } \psi_{1,2} \rightarrow V .
$$

Theorems II.3-II.6, III.1, III.7 provide useful formulae for the latter step, while for the former one can discretize the eigenvalue equation and call upon standard matrix solving packages. While we have no proof that the fixed point or points are attractive, preliminary numerical work on rectangular domains [10] seems to indicate that iteration of the mapping is convergent to a plausible optimizer. For large $M$, numerical instabilities arise because of the exponential smallness of the gap and the values of the eigenfunctions in the barrier, but, at least in one dimension, this can be compensated for by asymptotic analysis (cf. the asymptotics for $S_{p, \infty}^{\prime}$ as $M \rightarrow \infty$ in [9]).

Acknowledgments. The authors are grateful to Don Hinton, Glenn James, and Henry Simpson for useful discussions, and to Phil Schaefer for organizing the conference "Maximum Principles and Eigenvalue Problems in Partial Differential Equations," June, 1987, at the University of Tennessee.

\section{REFERENCES}

[1] M. S. Ashbaugh and R. D. Benguria, Optimal lower bounds for eigenvalue gaps for Schrödinger operators with symmetric single-well potentials and related results, pp. 134-145 in Maximum Principles and Eigenvalue Problems in Partial Differential Equations, Philip W. Schaefer, ed. New York: Wiley and Harlow, Essex: Longman 1988.

[2] M. S. Ashbaugh and E. M. Harrell II, Potentials having extremal eigenvalues subject to p-norm constraints, pp. 19-29 in Proceeding of the 1984 Workshop on Spectral Theory of Sturm-Liouville Differential Operators, Hans G. Kaper and A. Zettl, eds., ANL-84-73, Argonne, Illinois: Argonne National Laboratory 1984. (Available from National Technical Information Service, Springfield, Virginia.)

[3] _- Maximal and minimal eigenvalues and their associated nonlinear equations, J. Math. Phys., 28 (1987), 1770-1786.

[4] I. Chavel, Eigenvalues in Riemannian Geometry, New York: Academic, 1984. 
[5] R. Courant and D. Hilbert, Methods of Mathematical Physics, vol. I. New York: Wiley Interscience 1953.

[6] H. Egnell, Extremal Properties of the First Eigenvalue of a Class of Elliptic Eigenvalue Problems, Annali Scuol. Norm. Sup. Pisa, 1987.

[7] D. Gilbarg and N. S. Trudinger, Elliptic Partial Differential Equations of Second Order, second edition, Grundlehren der mathematischen Wissenschaften 224. Berlin, Heidelberg, New York, London, Paris, Tokyo: Springer-Verlag 1983.

[8] E. M. Harrell II, Hamiltonian operators with maximal eigenvalues, J. Math. Phys., 25 (1984), 48-51. Erratum, 27 (1986), 419.

[9] - General bounds for the eigenvalues of Schroedinger operators, pp. 146-166 in Maximum Principles and Eigenvalue Problems in Partial Differential Equations, Philip W. Schaefer, ed. New York: Wiley and Harlow, Essex: Longman 1988.

[10] E. M. Harrell II and G. E. James, work in preparation (numerical computations).

[11] E. M. Harrell II and R. Svirsky, Potentials producing maximally sharp resonances, Trans. Amer. Math. Soc., 293 (1986), 723-736.

[12] T. Kato, Perturbation Theory for Linear Operators, second edition, Grundlehren der mathematischen Wissenschaften 132. Berlin, Heidelberg, New York, Tokyo: Springer-Verlag 1976.

[13] W. Kirsch, On the difference between eigenvalues of Sturm-Liouville operators and the semiclassical limit, pp. 230-235 in Differential Equations and Mathematical Physics, I. W. Knowles and Y. Saitō, eds., Lecture Notes in Mathematics 1285. Berlin, Heidelberg, New York, London, Paris, Tokyo: Springer-Verlag 1987.

[14] W. Kirsch and B. Simon, Universal lower bounds on eigenvalue splittings for one dimensional Schrödinger operators, Comm. Math. Phys., 97 (1985), 453-460.

[15] _ Comparison theorems for the gap of Schrödinger operators, J. Funct. Anal., 75 (1987), 396-410.

[16] L. D. Landau and E. M. Lifshitz, Quantum Mechanics, Nonrelativistic Theory, Course of Theoretical Physics 3. Oxford, New York, Toronto, Sydney, Paris, Frankfurt: Pergamon, 1977.

[17] S. Nakamura, A remark on eigenvalue splittings for one-dimensional double-well Hamiltonians, Lett. Math. Phys., 11 (1986), 337-340.

[18] J. Pöschel and E. Trubowitz, Inverse Spectral Theory. New York: Academic 1986.

[19] M. H. Protter, Lower bounds for the spectrum of second order operators and systems of operators, pp. 82-93 in Maximum Principles and Eigenvalue Problems in Partial Differential Equations, Philip W. Schaefer, ed. New York: Wiley and Harlow, Essex: Longman 1988.

[20] M. Reed and B. Simon, Fourier Analysis, Self-Adjointness, Methods of Modern Mathematical Physics II. New York: Academic 1975.

[21] _ Analysis of Operators, Methods of Modern Mathematical Physics IV. New York: Academic 1978.

[22] W. Rudin, Real and Complex Analysis. New York, Toronto, and London: McGraw-Hill, 1966.

[23] I. M. Singer, B. Wong, S.-T. Yau, and S. S.-T. Yau, An estimate of the gap of the first two eigenvalues in the Schroedinger operator, Ann. Scuola Norm. Sup. Pisa (4), 12 (1985), 319-333.

[24] R. Svirsky, Maximally resonant potentials subject to p-norm constraints, Pacific J. Math., 129 (1987), 357-374. 
[25] Q. Yu and J.-Q. Zhong, Lower bounds of the gap between the first and second eigenvalues of the Schroedinger operator, Trans. Amer. Math. Soc., 294 (1986), 341-349.

Received December 28, 1988 and in revised form July 3, 1989. The first author was partially supported by a Summer Research Fellowship granted by the Research Council of the University of Missouri-Columbia. The second author was partially supported by USNSF grants DMS 8504354 and DMS 8801309.

UNIVERSITY OF MISSOURI

Columbia, MO 65211

Georgia Institute of Technology

AtLANTA, GA 30332-0160

AND

UNIVERSITY OF TENNESSEE

KNOXVILLE, TN 37916-1300 


\section{PACIFIC JOURNAL OF MATHEMATICS EDITORS}

V. S. VARADARAJAN

(Managing Editor)

University of California

Los Angeles, CA 90024-1555-05

Herbert Clemens

University of Utah

Salt Lake City, UT 84112

THOMAS ENRIGHT

University of California, San Diego

La Jolla, CA 92093

\section{R. FINN}

Stanford University

Stanford, CA 94305

HermanN FlaschKa

University of Arizona

Tucson, AZ 85721

VAUGHAN F. R. JoNES

University of California

Berkeley, CA 94720

STEVEn KeRCKHOFF

Stanford University

Stanford, CA 94305
C. C. MOORE

University of California

Berkeley, CA 94720

MARTIN SCHARLEMANN

University of California

Santa Barbara, CA 93106

Harold STARK

University of California, San Diego La Jolla, CA 92093

\section{ASSOCIATE EDITORS}

R. ARENS

E. F. BECKENBACH (1906-1982)
B. H. NeumanN

\section{SUPI}

UNIVERSITY OF ARIZONA

UNIVERSITY OF BRITISH COLUMBIA

CALIFORNIA INSTITUTE OF TECHNOLOGY

UNIVERSITY OF CALIFORNIA

MONTANA STATE UNIVERSITY

UNIVERSITY OF NEVADA, RENO

NEW MEXICO STATE UNIVERSITY

OREGON STATE UNIVERSITY
F. WolF
K. YoshidA
(1904-1989)

UNIVERSITY OF OREGON UNIVERSITY OF SOUTHERN CALIFORNIA

STANFORD UNIVERSITY

UNIVERSITY OF HAWAII

UNIVERSITY OF TOKYO

UNIVERSITY OF UTAH

WASHINGTON STATE UNIVERSITY

UNIVERSITY OF WASHINGTON 


\section{Pacific Journal of Mathematics}

Vol. 147, No. $1 \quad$ January, 1991

Mark S. Ashbaugh, Evans Malott Harrell, II and Roman Svirsky, On minimal and maximal eigenvalue gaps and their causes $\ldots \ldots \ldots \ldots \ldots 1$

Robert Coleman and Francis Oisin McGuinness, Rational formal group

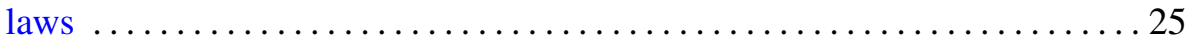

Jacek M. Cygan and Leonard Frederick Richardson, $D$-harmonic distributions and global hypoellipticity on nilmanifolds ...........29

Satya Deo and Kalathoor Varadarajan, Some examples of nontaut

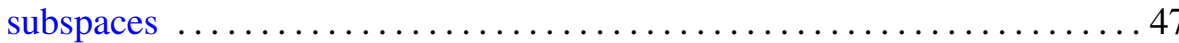

Maria Fragoulopoulou, Automatic continuity of *-morphisms between

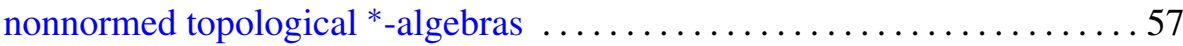

Stephen J. Gardiner, Removable singularities for subharmonic functions . . . 71 Herbert Paul Halpern, Victor Kaftal and László Zsidó, Finite weight projections in von Neumann algebras $\ldots \ldots \ldots \ldots \ldots \ldots \ldots \ldots \ldots \ldots$

Telemachos E. Hatziafratis, Explicit $\bar{\partial}$-primitives of Henkin-Leiterer kernels on Stein manifolds

Ka Hin Leung, A construction of an ordered division ring with a rank one

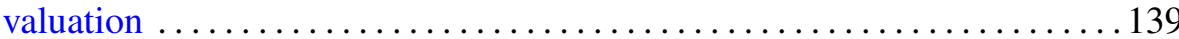

Christopher K. McCord, Nielsen numbers and Lefschetz numbers on solvmanifolds

Katsuro Sakai and Raymond Y. T. Wong, Manifold subgroups of the homeomorphism group of a compact $Q$-manifold

Caroline Perkins Sweezy, $L$-harmonic functions and the exponential square class 